\title{
Cineoltherapie bringt rasche Linderung
}

In randomisierten Doppelblindstudien $[1,2,3]$ wurden die klinische Wirksamkeit und die Verträglichkeit von Cineol bei akuter Sinusitis und Bronchitis sowie zur Zusatzbehandlung bei chronischen und entzündlichen Erkrankungen der Atemwege nachgewiesen. Die neue nicht-interventionelle, multizentrische Studie mit 336 Kindern und Kleinkindern mit akuter Bronchitis bestätigt den positiven klinischen Verlauf, Compliance und Verträglichkeit von Soledum ${ }^{\oplus}$ Kapseln junior.

Alle kleinen Patienten waren an einer akuten Bronchitis mit einem BronchitisSymptom-Summenscore (BSS) von über 5 erkrankt. Ein Fünftel der Patienten litt unter Fieber, Rhinitis und/oder Kopfschmerzen. Die kleinen Patienten wurden nach der Erstdiagnose sieben bis neun Tage lang mit dreimal täglich einer
Kapsel des Prüfpräparates behandelt, die 100 mg Cineol enthielt.

\section{Hohe Patientenakzeptanz}

Die Verträglichkeit des Prüfpräparates wurde sowohl von Ärzten als auch von Patienten zu 93\% als sehr gut und gut beurteilt. Bei einer sehr geringen Inzidenzrate von unter $1 \%$ klagten lediglich drei Patienten über Übelkeit, Erbrechen und Diarrhö. Die Patientenakzeptanz wurde von Therapeuten und Eltern mit 79\% in der jüngeren und $89 \%$ in der älteren Studiengruppe bewertet.

\section{Signifikante Besserung des BSS}

Die durchschnittliche Therapiedauer betrug in beiden Gruppen acht Tage. Der BSS verbesserte sich bereits nach einer drei- bis fünftägigen Behandlung signifikant. Er sank von durchschnittlich 8,6

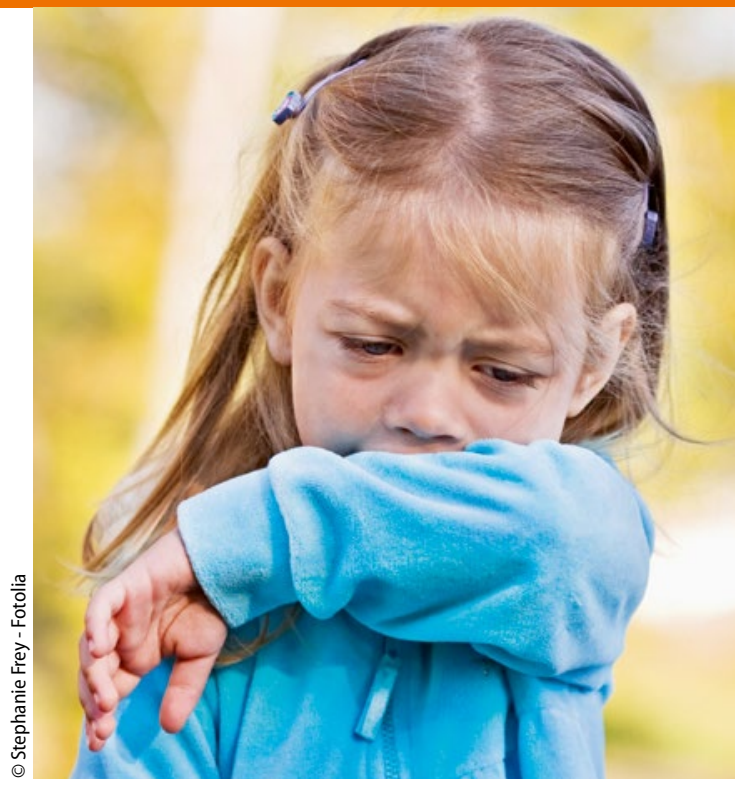

zu Therapiebeginn auf 5,1 nach drei bis fünf Tagen Behandlung. Nach sieben bis neun Tagen war er dann im Durchschnitt auf 1,4 gesunken. Zum Studienende waren $79 \%$ der Teilnehmer beschwerdefrei.

- Red.

1. Kehrl W et al. Laryngoscope. 2004;4:738-42

2. Tesche $S$ et al. Eur Arch Otorhinolaryngol. 2009;265:1355-9

3. Fischer J, Dethlefsen U. Cough. 2013;9:25-30

\section{HIV-Infektion}

\section{Frühzeitige Diagnose und Therapie verbessern Prognose}

_ Heutzutage kann eine HIV-Infektion bei fast allen Betroffenen effektiv eingedämmt werden, betonte PD Dr. Christian Hoffmann, Hamburg, „Wenn die Behandlung früh einsetzt, haben die Betroffenen eine annähernd normale Lebenserwartung.“

\section{Auf Indikatorerkrankungen achten}

Eine frühe Behandlung ist aber nur möglich, wenn die Diagnose möglichst rasch gestellt wird. Hier gebe es in Deutschland noch relevante Defizite, betonte Hoffmann. Er plädierte dafür, $\mathrm{Pa}$ tienten mit sogenannten Indikatorerkrankungen generell zu testen. $\mathrm{Zu}$ diesen gehören z. B. sexuell übertragbare Erkrankungen, Hepatitiden, maligne
Lymphome, zervikale oder anale Dysplasien, Herpes Zoster und eine unklare Leuko- oder Thrombopenie.

Den Nutzen einer frühen antiretroviralen Therapie hat die im Sommer 2015 publizierte START-Studie mit 4.700 HIVInfizierten gezeigt. In dieser Studie wurde die Effektivität eines Therapiebeginns bei CD4-Zellzahlen von über 500/ $\mu \mathrm{l}$ mit der eines späteren Behandlungsbeginns (CD4-Zellen < 350/ $\mu$ l) verglichen. Der primäre Endpunkt bestand aus AIDSdefinierenden Erkrankungen, Todesfällen sowie ernsten Nicht-AIDS-Ereignissen. Die früh behandelten Patienten schnitten dabei signifikant besser ab. „Wir sollten HIV-Infizierte heute so früh wie möglich behandeln“, so Hoffmann.
Dies unterstütze auch die Prävention, wie PD Dr. Christoph Wyen, Köln, betonte: „Wenn die Viruslast dauerhaft unter der Nachweisgrenze ist, sind die Betroffenen nicht mehr infektiös.“

\section{Patentfreie HIV-Präparate}

Aufgrund der Tatsache, dass immer mehr antiretrovirale Medikamente patentfrei werden, dürften auch die Kosten der antiretroviralen Therapien deutlich sinken. Das Unternehmen Hexal hält eine breite Produktpalette bereit, die neben häufiger eingesetzten auch seltener verordnete Präparate umfasst.

Red.

- Nach Informationen von Hexal 